УДК $517.55+512.64$

\title{
On a Quantum Logical Element Associated with the Radical of a Complex Matrix
}

\author{
Matvey E. Durakov* \\ Anton S. Kerp ${ }^{\dagger}$ \\ Anton S. Lukotkin $\ddagger$ \\ Institute of Mathematics and Computer Science \\ Siberian Federal University \\ Svobodny, 79, Krasnoyarsk, 660041
}

Russia

Received 14.08.2018, received in revised form 08.12.2018, accepted 20.02.2019

We construct new quantum logical elements realised by radicals of complex matrices. The geometry of these radicals is studied on the Reinhardt diagram.

Keywords: logical element, quantum computer, matrix radical, Reinhardt diagram.

DOI: $10.17516 / 1997-1397-2019-12-2-222-230$.

\section{Introduction}

Quantum Informatics is a new branch of science arising at the junction of quantum mechanics, algorithms and information theory. General principles and laws managing the dynamics of complex quantum systems are studied in quantum computer science. Quantum computer is a computing device based on the principles of quantum mechanics.

The idea of building a quantum computer was proposed in 1980 by the Soviet mathematician Yu. I. Manin [1]. This idea was supported by physicists, in particular, by P. Benioff [2] and a Nobel laureate R. Feynman [3].

The necessity for a quantum computer arises when we try to study difficult multiparticle systems, e.g. biological, using physical methods.

High hopes are placed on the perfection of this device, and active work is underway in this direction. In order to develop and apply the principles of quantum physics new research tools are required. It is a quantum computer that has become a modern device that makes it possible to study various phenomena and calculate the necessary data. So far, only a limited computer has been built, but this was enough to make sure that a full-fledged quantum computer is needed.

A quantum computer uses non-classical algorithms for computing that are realized by nonclassical logical elements.

A logical element is a computer device that performs one specific operation on input signals according to the rules of the algebra of logic. For example, a logical element reflecting transition to the negation is represented by the following scheme (Fig. 1):

\footnotetext{
*durakov_m_1997@mail.ru

†anton kerp@mail.ru

‡zgar4@yandex.ru

(c) Siberian Federal University. All rights reserved
} 


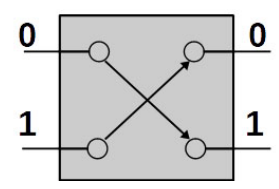

Fig. 1. A scheme of the logical element representing the negation

Since in logic the negation of a statement is formed with the help of the particle "not", the depicted element will be denoted by the symbol NOT.

Within the framework of the real analysis it is impossible to build a logical element $\sqrt{N O T}$, i.e. such an element, for which $\sqrt{N O T} \times \sqrt{N O T}=N O T$, where multiplication means the successive application of elements. In other words, even within the framework of the classical multi-valued logic based on classical probability theory, the equation is unsolvable. Let us show this.

A technical scheme reflecting this equation is presented in Fig. 2.

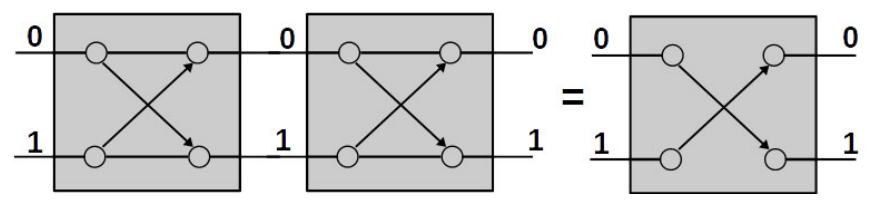

Fig. 2. A technical scheme for equation $\sqrt{N O T} \times \sqrt{N O T}=N O T$

Here on the left there are two copies of one (unknown) logical element $X$. It is assumed that transitions $0 \rightarrow 0,0 \rightarrow 1,1 \rightarrow 0,1 \rightarrow 1$ occur with probabilities $P_{00}, P_{01}, P_{10}, P_{11}$ respectively (see Fig. 3).

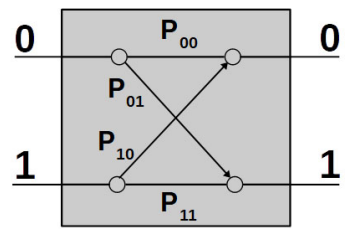

Fig. 3. A scheme of the logical element $X$

For the element «NOT» we have: $P_{00}=P_{11}=0 ; P_{01}=P_{10}=1$. Therefore, to satisfy the equality $X \times X=N O T$ we obtain the system of equations

$$
\left\{\begin{array}{l}
P_{00} P_{00}+P_{01} P_{10}=0, \\
P_{00} P_{01}+P_{01} P_{11}=1, \\
P_{10} P_{00}+P_{11} P_{10}=1, \\
P_{11} P_{11}+P_{10} P_{01}=0 .
\end{array}\right.
$$

Since $P_{i j} \geqslant 0$, then from the first and fourth equations we obtain $P_{00}=P_{11}=0$, therefore, the second and third equations are reduced to $0=1$. Thus, this scheme is unrealizable for real non-negative values $P_{i j}$.

The way to the realization of the considered equality is suggested by quantum mechanics, in which the amplitude of the transition probability $i \rightarrow j$ is a complex number $c_{i j}$, for which $P_{i j}=\left|c_{i j}\right|^{2}$. Thus, if in the given system of equations we replace $P_{i j}$ by complex numbers $c_{i j}$, 
then one should consider the equations:

$$
\begin{aligned}
& P(0 \rightarrow 0)=\left|c_{00} c_{00}+c_{01} c_{10}\right|^{2}, \\
& P(0 \rightarrow 1)=\left|c_{00} c_{01}+c_{01} c_{11}\right|^{2}, \\
& P(1 \rightarrow 0)=\left|c_{10} c_{00}+c_{11} c_{10}\right|^{2}, \\
& P(1 \rightarrow 1)=\left|c_{11} c_{11}+c_{10} c_{01}\right|^{2} .
\end{aligned}
$$

Now consider the system of equations:

$$
\left\{\begin{array}{l}
0=\left|c_{00} c_{00}+c_{01} c_{10}\right|^{2}, \\
1=\left|c_{00} c_{01}+c_{01} c_{11}\right|^{2}, \\
1=\left|c_{10} c_{00}+c_{11} c_{10}\right|^{2}, \\
0=\left|c_{11} c_{11}+c_{10} c_{01}\right|^{2} .
\end{array}\right.
$$

Some examples of the roots of this system of equations were presented in the book of Guts [4]: $c_{00}=c_{11}=i / \sqrt{2} ; c_{01}=e^{-i \alpha} / \sqrt{2} ; c_{10}=e^{i \alpha} / \sqrt{2}$.

From the main results of this article, it follows that all complex solutions of the system (1) are parametrized in the following form with 2 parameters $\alpha, \beta \in[0,2 \pi]$ :

$$
c_{00}=c_{11}=e^{i \alpha} / \sqrt{2} ; \quad c_{01}=e^{i \beta} / \sqrt{2} ; \quad c_{10}=e^{i(\pi+2 \alpha-\beta)} / \sqrt{2} .
$$

In fact, the problem of finding a logical element $\sqrt{N O T}$ is reduced to finding a matrix whose square is equal to

$$
N O T=\left(\begin{array}{cc}
0 & e^{i(\alpha-\beta)} \\
e^{i(\alpha+\beta)} & 0
\end{array}\right), \text { where } \quad \alpha, \beta \in \mathbb{R} .
$$

This way of representing the matrix NOT allows us to write the equation $X^{2}=N O T$ as a matrix equation:

$$
\left(\begin{array}{ll}
c_{00} & c_{01} \\
c_{10} & c_{11}
\end{array}\right)^{2}=\left(\begin{array}{ll}
c_{00} c_{00}+c_{01} c_{10} & c_{00} c_{01}+c_{01} c_{11} \\
c_{10} c_{00}+c_{11} c_{10} & c_{11} c_{11}+c_{10} c_{01}
\end{array}\right)=\left(\begin{array}{cc}
0 & e^{i(\alpha-\beta)} \\
e^{i(\alpha+\beta)} & 0
\end{array}\right) .
$$

The aim of this paper is to find new non-classical logical elements. Namely, we investigate the problem of finding $\sqrt[n]{N O T}$, that is, the problem of finding all solutions of the equation $X^{n}=$ NOT.

First, we note that the successive application $n$ times of a non-classical element $X$ corresponds to raising to the $n$-th degree of a complex matrix $C=\left(\begin{array}{ll}c_{00} & c_{01} \\ c_{10} & c_{11}\end{array}\right)$, that is $X^{n}$ corresponds $C^{n}$.

Theorem 1. All solutions of the matrix equation $X^{n}=$ NOT are given by matrices

$$
\left(\begin{array}{ll}
c_{00} & c_{01} \\
c_{10} & c_{11}
\end{array}\right)=\left(\begin{array}{cc}
\frac{t_{j} e^{i \frac{\alpha+2 \pi k}{n}}}{1+t_{j}} & \frac{e^{i\left(\frac{\alpha+2 \pi k}{n}-\beta\right)}}{1+t_{j}} \\
\frac{e^{i\left(\frac{\alpha+2 \pi k}{n}+\beta\right)}}{1+t_{j}} & \frac{t_{j} e^{i \frac{\alpha+2 \pi k}{n}}}{1+t_{j}}
\end{array}\right), \alpha, \beta \in \mathbb{R}
$$

where $k, j=0, \ldots, n-1$, and $t_{j}$ are the solutions of the following algebraic equation:

$$
\sum_{k=0}^{\left\lfloor\frac{n}{2}\right\rfloor} C_{n}^{2 k} t^{n-2 k}=0 .
$$

In Section 2 we calculate all roots $t_{j}$ of the equation (3) involved in the formulation of this theorem. 


\section{Proof of Theorem 1}

We write the matrix $X$ in the form

$$
X=\left(\begin{array}{ll}
c_{00} & c_{01} \\
c_{10} & c_{11}
\end{array}\right) .
$$

In order to raise $X$ to $n$-th degree, we reduce it to the diagonal form. The characteristic roots of the matrix $X$ are the following:

$$
\lambda_{1}=\frac{c_{00}+c_{11}+\sqrt{D}}{2}, \quad \lambda_{2}=\frac{c_{00}+c_{11}-\sqrt{D}}{2},
$$

where $D$ is the discriminant of the characteristic equation, equal to

$$
D=\left(c_{00}+c_{11}\right)^{2}-4\left(c_{00} c_{11}-c_{01} c_{10}\right) .
$$

The diagonal matrix

$$
V=\left(\begin{array}{cc}
\lambda_{1} & 0 \\
0 & \lambda_{2}
\end{array}\right)
$$

is related to $X$ by the equality $V S=S X$, where $S=\left(\begin{array}{ll}s_{00} & s_{01} \\ s_{10} & s_{11}\end{array}\right)$ is a transform matrix. This leads to the following result:

$$
S=\left(\begin{array}{cc}
\frac{c_{11}-c_{00}+\sqrt{D}}{2 \sqrt{D}} & \frac{-c_{01}}{\sqrt{D}} \\
\frac{c_{00}-c_{11}+\sqrt{D}}{2 c_{01}} & 1
\end{array}\right), \quad S^{-1}=\left(\begin{array}{cc}
1 & \frac{c_{01}}{\sqrt{D}} \\
\frac{c_{11}-c_{00}-\sqrt{D}}{2 c_{01}} & \frac{c_{11}-c_{00}+\sqrt{D}}{2 \sqrt{D}}
\end{array}\right)
$$

Consider the matrix $X^{n}$ first for even $n$, writing it as

$$
X^{n}=\left(\begin{array}{ll}
x_{00} & x_{01} \\
x_{10} & x_{11}
\end{array}\right) .
$$

From the relationship $X^{n}=S^{-1} V^{n} S$ we get the expressions for $x_{i j}$ :

$$
\left\{\begin{array}{l}
x_{00}=\frac{1}{2^{n}}\left(\sum_{k=0}^{\frac{n}{2}-1}\left[\left(c_{00}+c_{11}\right)^{n-2 k-1} D^{k}\left(C_{n}^{2 k+1}\left(c_{00}-c_{11}\right)+C_{n}^{2 k}\left(c_{00}+c_{11}\right)\right)\right]+D^{\frac{n}{2}}\right), \\
x_{01}=\frac{c_{01}}{2^{n-1}} \sum_{k=1}^{\frac{n}{2}}\left[C_{n}^{2 k-1}\left(c_{00}+c_{11}\right)^{n-2 k+1} D^{k-1}\right], \\
x_{10}=\frac{c_{10}}{2^{n-1}} \sum_{k=1}^{\frac{n}{2}}\left[C_{n}^{2 k-1}\left(c_{00}+c_{11}\right)^{n-2 k+1} D^{k-1}\right], \\
x_{11}=\frac{1}{2^{n}}\left(\sum_{k=0}^{\frac{n}{2}-1}\left[\left(c_{00}+c_{11}\right)^{n-2 k-1} D^{k}\left(C_{n}^{2 k+1}\left(c_{11}-c_{00}\right)+C_{n}^{2 k}\left(c_{00}+c_{11}\right)\right)\right]+D^{\frac{n}{2}}\right) .
\end{array}\right.
$$

Considering the equality $X^{n}=N O T$, we arrive at the following system of equations:

$$
\left\{\begin{array}{l}
\frac{1}{2^{n}}\left(\sum_{k=0}^{\frac{n}{2}-1}\left[\left(c_{00}+c_{11}\right)^{n-2 k-1} D^{k}\left(C_{n}^{2 k+1}\left(c_{00}-c_{11}\right)+C_{n}^{2 k}\left(c_{00}+c_{11}\right)\right)\right]+D^{\frac{n}{2}}\right)=0, \\
\frac{c_{01}}{2^{n-1}} \sum_{k=1}^{\frac{n}{2}}\left[C_{n}^{2 k-1}\left(c_{00}+c_{11}\right)^{n-2 k+1} D^{k-1}\right]=e^{i(\alpha-\beta)}, \\
\frac{c_{10}}{2^{n-1}} \sum_{k=1}^{\frac{n}{2}}\left[C_{n}^{2 k-1}\left(c_{00}+c_{11}\right)^{n-2 k+1} D^{k-1}\right]=e^{i(\alpha+\beta)}, \\
\frac{1}{2^{n}}\left(\sum_{k=0}^{\frac{n}{2}-1}\left[\left(c_{00}+c_{11}\right)^{n-2 k-1} D^{k}\left(C_{n}^{2 k+1}\left(c_{11}-c_{00}\right)+C_{n}^{2 k}\left(c_{00}+c_{11}\right)\right)\right]+D^{\frac{n}{2}}\right)=0 .
\end{array}\right.
$$


Subtracting the fourth equation from the first one, we get:

$$
\left(c_{00}-c_{11}\right) \frac{1}{2^{n-1}} \sum_{k=1}^{\frac{n}{2}}\left[C_{n}^{2 k-1}\left(c_{00}+c_{11}\right)^{n-2 k+1} D^{k-1}\right]=0 .
$$

Since the multiplier represented as a sum is not equal to zero (in view of the second equation of the system), we obtain $c_{00}=c_{11}$. Note that in this case $D=4 c_{01} c_{10}$. Making the appropriate substitution, our system will take the form:

$$
\left\{\begin{array}{l}
\sum_{k=0}^{\frac{n}{2}} C_{n}^{2 k} c_{00}^{n-2 k}\left(c_{01} c_{10}\right)^{k}=0 \\
c_{01} \sum_{k=1}^{\frac{n}{2}} C_{n}^{2 k-1} c_{00}^{n-2 k+1}\left(c_{01} c_{10}\right)^{k-1}=e^{i(\alpha-\beta)}, \\
c_{10} \sum_{k=1}^{\frac{n}{2}} C_{n}^{2 k-1} c_{00}^{n-2 k+1}\left(c_{01} c_{10}\right)^{k-1}=e^{i(\alpha+\beta)}, \\
\sum_{k=0}^{\frac{n}{2}} C_{n}^{2 k} c_{00}^{n-2 k}\left(c_{01} c_{10}\right)^{k}=0 .
\end{array}\right.
$$

Here, the first and fourth equations are the same, so we should consider a system of three equations. Dividing the third equation by the second, we obtain the relation $c_{10}=c_{01} e^{2 i \beta}$, according to which the system look like:

$$
\left\{\begin{array}{l}
\sum_{k=0}^{\frac{n}{2}} C_{n}^{2 k} c_{00}^{n-2 k}\left(c_{01} e^{i \beta}\right)^{2 k}=0 \\
c_{01} \sum_{k=1}^{\frac{n}{2}} C_{n}^{2 k-1} c_{00}^{n-2 k+1}\left(c_{01} e^{i \beta}\right)^{2 k-2}=e^{i(\alpha-\beta)} \\
\sum_{k=1}^{\frac{n}{2}} C_{n}^{2 k-1} c_{00}^{n-2 k+1}\left(c_{01} e^{i \beta}\right)^{2 k-1}=e^{i \alpha}
\end{array}\right.
$$

Note that the third equation is the second one multiplied by $e^{i \beta}$, so the second equation can be ignored. Adding the left parts of the remaining equation, we get

$$
\sum_{k=1}^{\frac{n}{2}} C_{n}^{2 k-1} c_{00}^{n-2 k+1}\left(c_{01} e^{i \beta}\right)^{2 k-1}+\sum_{k=0}^{\frac{n}{2}} C_{n}^{2 k} c_{00}^{n-2 k}\left(c_{01} e^{i \beta}\right)^{2 k}=\left(c_{00}+c_{01} e^{i \beta}\right)^{n}
$$

As a result, we obtain a system of two equations:

$$
\left\{\begin{array}{l}
\left(c_{00}+c_{01} e^{i \beta}\right)^{n}=e^{i \alpha}, \\
\sum_{k=0}^{\frac{n}{2}} C_{n}^{2 k} c_{00}^{n-2 k}\left(c_{01} e^{i \beta}\right)^{2} k=0 .
\end{array}\right.
$$

For convenience, rewrite this system in the following form, making replacements $z=c_{00}$ and $w=c_{01} e^{i \beta}$ :

$$
\left\{\begin{array}{l}
(z+w)^{n}=e^{i \alpha} \\
\sum_{k=0}^{\frac{n}{2}} C_{n}^{2 k} z^{n-2 k} w^{2 k}=0
\end{array}\right.
$$

After another notation $t=\frac{z}{w}$, it is written as

$$
\left\{\begin{array}{l}
(w(1+t))^{n}=e^{i \alpha} \\
\sum_{k=0}^{\frac{n}{2}} C_{n}^{2 k} t^{n-2 k}=0
\end{array}\right.
$$


From the first equation we get that $w=\frac{e^{i \frac{\alpha+2 \pi k}{n}}}{1+t}$ and, therefore, $z=\frac{t e^{i \frac{\alpha+2 \pi k}{n}}}{1+t}$. Thus, we get:

$$
c_{00}=c_{11}=z=\frac{t_{j} e^{i \frac{\alpha+2 \pi k}{n}}}{1+t_{j}}, \quad c_{01}=\frac{w}{e^{i \beta}}=\frac{e^{i \frac{\alpha+2 \pi k}{n}-\beta}}{1+t_{j}}, \quad c_{10}=\frac{w}{e^{i \beta}} e^{2 i \beta}=\frac{e^{i \frac{\alpha+2 \pi k}{n}+\beta}}{1+t_{j}} .
$$

Similar reasoning for odd $n$ leads to the following system:

$$
\left\{\begin{array}{l}
(w(1+t))^{n}=e^{i \alpha} \\
\sum_{k=0}^{\frac{n-1}{2}} C_{n}^{2 k} t^{n-2 k}=0
\end{array}\right.
$$

Since

$$
\left\lfloor\frac{n}{2}\right\rfloor= \begin{cases}\frac{n}{2} & \text { if } n \text { is even } \\ \frac{n-1}{2} & \text { if } n \text { is odd }\end{cases}
$$

one gets the system:

$$
\begin{cases}(w(1+t))^{n}=e^{i \alpha}, & n \in \mathbb{N}, \\ \sum_{k=0}^{\left.\frac{n-1}{2}\right\rfloor} C_{n}^{2 k} t^{n-2 k}=0, & n \in \mathbb{N} .\end{cases}
$$

That is what we wanted to prove.

\section{Roots of the equation (3)}

Theorem 2. All roots $t=t_{j}$ of the equation

$$
\sum_{k=0}^{\left\lfloor\frac{n}{2}\right\rfloor} C_{n}^{2 k} t^{n-2 k}=0
$$

are exhausted by the set: $\left\{i \operatorname{ctg}\left(\frac{\pi+2 \pi j}{2 n}\right)\right\}_{j=0}^{n-1}$.

Proof. Note that the original equation is equivalent to

$$
\frac{(t+1)^{n}+(t-1)^{n}}{2}=0
$$

Indeed, the left part of the original equality can be represented by Newton's binomial formula for $(t+1)^{n}$ with missing monomials $\left\{C_{n}^{1} t^{n-1}, C_{n}^{3} t^{n-3}, \ldots\right\}$, that arise with a minus sign in the binomial decomposition for $(t-1)^{n}$.

Obviously, the solutions $t$ of the equation (4) satisfy the condition $|t+1|=|t-1|$, and therefore they are purely imaginary: $t=i b$. By substituting in (4) we arrive at the equation for $b$ :

$$
\left(\frac{i b+1}{i b-1}\right)^{n}=-1
$$

whence

$$
\frac{i b+1}{i b-1}=e^{i \frac{\pi+2 \pi j}{n}}, j=0,1, \ldots, n-1 .
$$


Solving each equation with respect to $b$, we obtain:

$$
b=b_{j}=i \frac{1+e^{i \frac{\pi+2 \pi j}{n}}}{1-e^{i \frac{\pi+2 \pi j}{n}}}=i \frac{e^{-i \frac{\pi+2 \pi j}{2 n}}+e^{i \frac{\pi+2 \pi j}{2 n}}}{e^{-i \frac{\pi+2 \pi j}{2 n}}-e^{i \frac{\pi+2 \pi j}{2 n}}}=-\operatorname{ctg}\left(\frac{\pi+2 \pi j}{2 n}\right) .
$$

Note that the function $-\operatorname{ctg} \phi$ is odd with respect to the center $\phi=\frac{\pi}{2}$ of the interval $(0, \pi)$. Because the values $\frac{\pi+2 \pi j}{2 n}$ are symmetrically located relative to $\phi=\frac{\pi}{2}$, we obtain that the set of numbers

$$
b_{j}=-\operatorname{ctg}\left(\frac{\pi+2 \pi j}{2 n}\right), j=0,1, \ldots, n-1
$$

coincides with the set

$$
b_{j}=\operatorname{ctg}\left(\frac{\pi+2 \pi j}{2 n}\right)=: \operatorname{ctg} \phi_{j}, j=0,1, \ldots, n-1 .
$$

As a result $t_{j}=i b_{j}=i c t g\left(\frac{\pi+2 \pi j}{2 n}\right), j=0, \ldots, n-1$, and by the fundamental theorem of algebra, these roots exhaust all zeros of the original polynomial of degree $n$.

Corollary. All solutions of the matrix equation $X^{n}=$ NOT are exhausted by the following set of $n^{2}$ matrices:

$$
\left(\begin{array}{cc}
\frac{i \operatorname{ctg}\left(\frac{\pi+2 \pi j}{2 n}\right) e^{i \frac{\alpha+2 \pi k}{n}}}{1+i \operatorname{ctg}\left(\frac{\pi+2 \pi j}{2 n}\right)} & \frac{e^{i\left(\frac{\alpha+2 \pi k}{n}-\beta\right)}}{1+i \operatorname{ctg}\left(\frac{\pi+2 \pi j}{2 n}\right)} \\
\frac{e^{i\left(\frac{\alpha+2 \pi k}{n}+\beta\right)}}{1+i \operatorname{ctg}\left(\frac{\pi+2 \pi j}{2 n}\right)} & \frac{i \operatorname{ctg}\left(\frac{\pi+2 \pi j}{2 n}\right) e^{i \frac{\alpha+2 \pi k}{n}}}{1+i \operatorname{ctg}\left(\frac{\pi+2 \pi j}{2 n}\right)}
\end{array}\right),
$$

where $k, j=0, \ldots, n-1$.

\section{Distribution of matrices $\sqrt[n]{N O T}$ on the Reinhardt diagram}

Consider the absolute values of the matrix elements described in the corollary:

$$
\begin{aligned}
& \left|c_{01}\right|=\left|c_{10}\right|=\left|\frac{1}{1+t_{j}}\right|=\left|\frac{1}{1+i c t g \phi_{j}}\right|=\frac{1}{\sqrt{1+c t g^{2} \phi_{j}}}=\frac{1}{\sqrt{\frac{1}{\sin ^{2} \phi_{j}}}}=\left|\sin \phi_{j}\right|, \\
& \left|c_{00}\right|=\left|c_{11}\right|=\left|\frac{t_{j}}{1+t_{j}}\right|=\left|\frac{i c t g \phi_{j}}{1+i c t g \phi_{j}}\right|=\frac{\left|c t g \phi_{j}\right|}{\sqrt{\frac{1}{\sin ^{2} \phi_{j}}}}=\left|c t g \phi_{j}\right|\left|\sin \phi_{j}\right|=\left|\cos \phi_{j}\right| .
\end{aligned}
$$

This shows that if one puts the modules $\left|c_{01}\right|=\left|c_{10}\right|$ on the ordinate axis, and the modules $\left|c_{00}\right|=\left|c_{11}\right|$ on the abscissa axis, then these modules will be uniformly distributed on the arc of the unit circle. The specified coordinate system for the modules is called the Reinhardt diagram (see [5]).

The Reinhardt diagram (Fig. 4) shows the modules for values $n=5$ and $n=50$.

Given the formula (2), for each $n \in \mathbb{N}$ the set of solutions of the equation $X^{n}=N O T$ is a family of

$$
\left\lceil\frac{n}{2}\right\rceil= \begin{cases}\frac{n}{2} & \text { if } n \text { is even } \\ \frac{n+1}{2} & \text { if } n \text { is odd }\end{cases}
$$


real two-dimensional tori. These tori are embedded in a 5-dimensional variety:

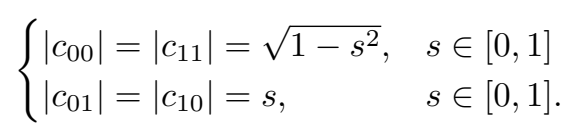

This variety coincides with the closure of the set of solutions of all equations $X^{n}=N O T$, $n=2,3, \ldots$.

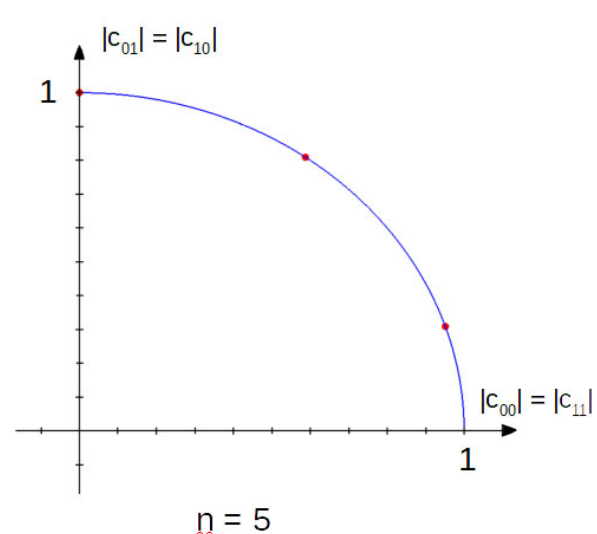

$n=5$

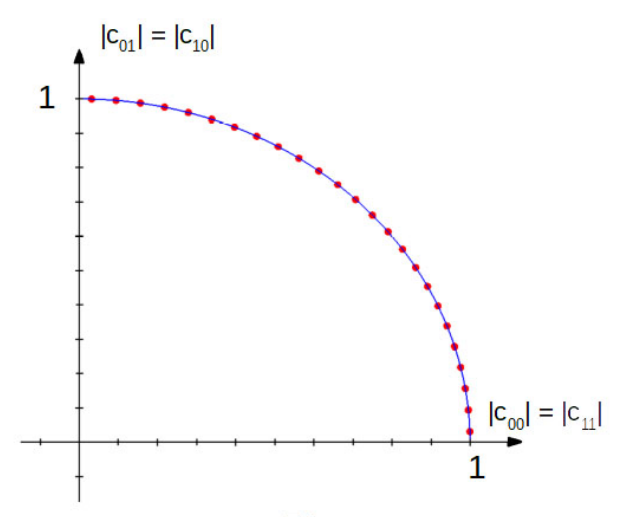

$n=50$

Fig. 4. Reinhardt diagram

\section{Comparison with the method described in the book of Gantmacher}

The Gantmacher book [6] presents an algorithm for finding all roots of $n$-th degree of a nondegenerate matrix. We shall apply it to our matrix NOT and compare the results.

Following [6], we reduce this matrix to the Jordan normal form $J$, find the transformation matrix $S$ and the inverse $S^{-1}$, that is, we represent our matrix as $N O T=S J S^{-1}$. We have:

$$
N O T=\left(\begin{array}{cc}
0 & e^{i(\alpha-\beta)} \\
e^{i(\alpha+\beta)} & 0
\end{array}\right) ; J=\left(\begin{array}{cc}
e^{i \alpha} & 0 \\
0 & -e^{i \alpha}
\end{array}\right), S=\left(\begin{array}{cc}
1 & -1 \\
e^{i \beta} & e^{i \beta}
\end{array}\right), S^{-1}=\left(\begin{array}{cc}
1 / 2 & e^{-i \beta} / 2 \\
-1 / 2 & e^{-i \beta} / 2
\end{array}\right) .
$$

Now find the root of $n$-th degree of the matrix $J$

$$
\sqrt[n]{J}=\left(\begin{array}{cc}
e^{\frac{i(\alpha+2 \pi k)}{n}} & 0 \\
0 & e^{\frac{i(\alpha+\pi+2 \pi k)}{n}}
\end{array}\right)
$$

Then all solutions of the original equation are the following $n^{2}$ matrices:

$$
X=S \sqrt[n]{J} S^{-1}=\left(\begin{array}{cc}
\frac{e^{i \frac{\alpha}{n}}\left(e^{i \frac{2 \pi k}{n}}+e^{i \frac{\pi+2 \pi j}{n}}\right)}{2} & \frac{e^{i\left(\frac{\alpha}{n}-\beta\right)}\left(e^{i \frac{2 \pi k}{n}}-e^{i \frac{\pi+2 \pi j}{n}}\right)}{2} \\
\frac{e^{i\left(\frac{\alpha}{n}+\beta\right)\left(e^{i \frac{2 \pi k}{n}}-e^{i \frac{\pi+2 \pi j}{n}}\right)}}{2} & \frac{e^{i \frac{\alpha}{n}}\left(e^{i \frac{2 \pi k}{n}}+e^{i \frac{\pi+2 \pi j}{n}}\right)}{2}
\end{array}\right),
$$

where $k, j=0, \ldots, n-1$.

To see the equivalence of these results with the results obtained above, it is sufficient to use the equality: $\operatorname{ctg}(x)=\frac{\cos (x)}{\sin (x)}=i \frac{e^{i x}+e^{-i x}}{e^{i x}-e^{-i x}}$. 
The first author was supported by a grant of the Ministry of Science and Higher Education of the Russian Federation (state task for Siberian Federal University no. 1.1462.2014/k).

\section{References}

[1] Yu.I.Manin, Computable and Uncomputable, Moscow, Sovetskoe radio, 1980 (in Russian).

[2] P.Benioff, Quantum mechanical hamiltonian models of turing machines, Journal of Statistical Physics, 29(1982), no. 3, 515-546.

[3] R.P.Feynman, Simulating physics with computers, International Journal of Theoretical Physics, (1982), no. 6, 467-488.

[4] A.K.Guts, Complex analysis and informatics, Omsk Gos. Univer., 2002 (in Russian).

[5] B.V.Shabat, Introduction to complex analysis part 2, Moscow, Nauka, 1976 (in Russian).

[6] F.R.Gantmacher, The Theory of Matrices, AMS Chelsea Publishing, Reprinted by American Mathematical Society, 2000.

\section{О квантовом логическом элементе, ассоциированным с радикалом комплексной матрицы}

Матвей Е. Дураков

Антон С. Керп

Антон С. Лукоткин

Институт математики и фундаментальной информатики

Сибирский федеральный университет Свободный, 79, Красноярск, 660041

Россия

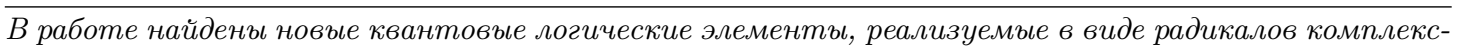
ных матрии, а также изучена их геометрия на диаграмме Рейнхардта.

Ключевые слова: логический элемент, квантовый компъютер, радикал матрицы, диаграмма Рейнхардта. 